\title{
ICE VELOCITY MAPPING USING TOPS SAR DATA AND OFFSET TRACKING
}

\author{
Jørgen Dall $^{(1)}$, Anders Kusk ${ }^{(1)}$, Ulrik Nielsen ${ }^{(1)}$, John Peter Merryman Boncori ${ }^{(2)}$ \\ (1) Technical University of Denmark, Ørsteds Plads 348, DK-2800 Kongens Lyngby, Denmark, jd@space.dtu.dk \\ (2) Instituto Nazionale de Geofisica e Vulcanologia, Via di Vigna Murata 605, \\ 00143 Roma, Italy,john.merryman@ingv.it
}

\begin{abstract}
Feature tracking and speckle tracking, are robust techniques to measure the velocity of glaciers and ice sheets. Displacement maps based on TOPS data may have small gaps if the bursts are not handled properly. Ice moving from one burst to a consecutive burst between two observations is not observed under the same squint angle, and hence speckle tracking is supposed to fail when cross-correlating consecutive bursts, whereas feature tracking provides the same result as when cross-correlating corresponding bursts. The size of the potential gaps depends on the ice displacement and the choice of Sentinel-1 product, as consecutive bursts overlap in the SLC product but not in the GRD product. An analysis of Sentinel-1 data from Greenland confirms the results expected from theory.
\end{abstract}

\section{INTRODUCTION}

Mapping the velocity fields of the continental ice sheets and their outlet glaciers is important in order to monitor and model the response of the cryosphere to global climate change. Since the mid 1990s, space-based SAR data have enabled measurement of ice velocities on a continental scale [1]. Compared to interferometry, offset tracking techniques excel in terms of robustness and ease of automation. Offset tracking estimates the range and azimuth displacements by determining the position of the peak resulting when cross-correlating two ice patches mapped with a temporal separation, typically between one day and one month. The term 'offset tracking' here refers to a class of methods including feature tracking [2][3] and speckle tracking [4][5]. The latter does not call for surface features like glacier crevasses, but relies on a stable speckle pattern, which in turn requires some degree of coherence. Speckle tracking can be applied to complex or detected data.

ESA's Sentinel-1A satellite was launched on 3 April 2014, and it carries a C-band synthetic aperture radar (SAR). Sentinel-1A represents a new generation of SAR satellites. Compared with the ERS and ASAR SARs, Sentinel-1A excels by a revisit time of just 12 days without sacrificing coverage. It can be operated in four different acquisition modes [6]:

- The Stripmap (SM) mode provides a $5 \mathrm{~m}$ by $5 \mathrm{~m}$ resolution over a $80 \mathrm{~km}$ wide swath.
- The Interferometric Wide Swath (IW) mode provides a $5 \mathrm{~m}$ by $20 \mathrm{~m}$ resolution over a $250 \mathrm{~km}$ wide swath.

- The Extra-Wide Swath (EW) mode provides a $20 \mathrm{~m}$ by $40 \mathrm{~m}$ resolution over a $400 \mathrm{~km}$ wide swath.

- The Wave Mode (WV) provides $5 \mathrm{~m}$ by $5 \mathrm{~m}$ resolution over $20 \mathrm{~km}$ by $20 \mathrm{~km}$ wave imagettes separated by $100 \mathrm{~km}$.

The IW and EW modes apply the Terrain Observation with Progressive Scan (TOPS) technique to obtain a wide swath at the expense of a coarser azimuth resolution [7]. The IW and EW swaths are composed of 3 and 5 slightly overlapping sub-swaths, respectively. Like ScanSAR, TOPS electronically scans the antenna beam in the elevation plane. However, unlike ScanSAR, TOPS also scans the antenna beam in azimuth. While a burst of pulses are transmitted and received from a sub-swath, the antenna beam is steered from backward to forward, as shown in Fig. 1.

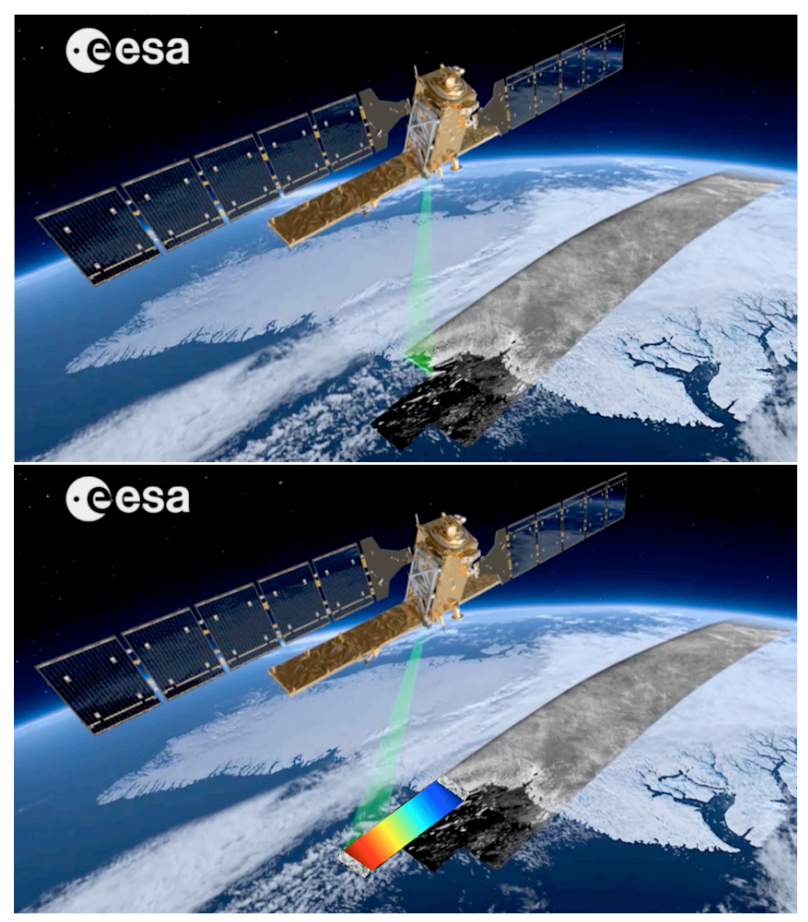

Figure 1. Sentinel-1 IW data acquisition. The Doppler centroid is negative (blue) at the beginning of the burst (top) and positive (red) at the end of it (bottom). (Figure based on [8]). 
The IW and EW acquisition modes support interferometry by synchronizing the bursts from pass to pass [9] such that they are spatially aligned. For stationary scenes this implies that also the Doppler spectra are aligned.

In the Ground Range Detected (GRD) Sentinel-1 product, bursts do not overlap, and the location of the burst seam is geographically fixed for all passes. In the Single Look Complex (SLC) product, bursts do overlap. SLC data are provided in slices of 25 seconds corresponding to about 9 bursts covering about $170 \mathrm{~km}$ in azimuth. The duration of focused bursts is 2.75 seconds corresponding to $19 \mathrm{~km}$ in azimuth. The burst overlaps in the near range swath, mid range swath, and far range swath are $405 \mathrm{~m}, 230 \mathrm{~m}$, and $180 \mathrm{~m}$, respectively [10].

This paper addresses issues that arise when TOPS data are used for offset tracking and the ice displacement between the two data acquisitions is comparable with (or exceeds) the azimuth dimension of the patches that are used for offset tracking. These issues are only relevant for IW and EW data. In Section 2 the problem is presented. In Section 3 the data analysis method is outlined, and in Section 4 the results are provided and discussed. Finally, in Section 5 the conclusions are made.

\section{PROBLEM}

The TOPS azimuth antenna scan implies that the Doppler centroid varies with the azimuth position within a burst. This Doppler centroid variation complicates TOPS SAR interferometry [11], in particular for non-stationary scenes, e.g. when measuring the velocity of glaciers and ice sheets [12]. Speckle tracking is less impacted than interferometry, as the phase difference between the two data sets is not exploited. Still, TOPS speckle tracking calls for an azimuth phase deramping and potentially an adaptive azimuth common band filtering [12].

TOPS speckle tracking has been thoroughly addressed by Scheiber et al. [12] assuming that corresponding bursts are combined. In the case of large displacements, and in particular when using GRD data, consecutive burst may be combined. This is addressed in this paper. Fig. 2 shows two consecutive bursts for each of two data acquisitions. In the GRD data, ice is crossing the fixed seam between the two bursts, and patches in one burst from the first data acquisition is cross-correlated with patches in the following patch from the second data acquisition. Consequently the two patches have very different Doppler centroids.

The difference in Doppler centroid is irrelevant for (pure) feature tracking, where the peak of the crosscorrelation function is ensured by glacier crevasses, melt ponds and other features. (The backscatter patterns

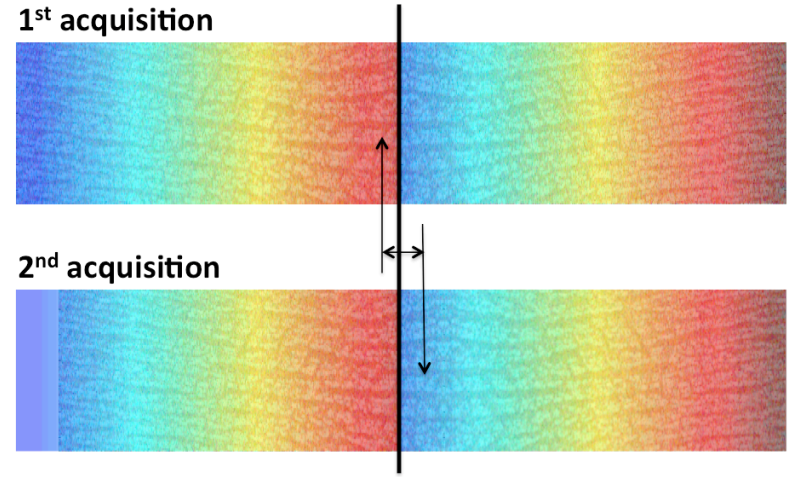

Figure 2. Ice moving from one burst to a consecutive burst between the two data acquisitions. The colours illustrate that the Doppler centroid changes from negative (blue) at the burst start (left) to positive (red) at the burst end right. The GRD product is assumed.

of the ice and the ice features are assumed not to vary significantly within the range of squint angles.) The speckle pattern, however, will change completely if a patch is observed with the antenna steered backward in one data acquisition and forward in the other acquisition, because the range of steering angles exceeds the range of squint angles under which a single target is observed. Equivalently, the variation of the Doppler centroid $f_{d c}$ exceeds the azimuth bandwidth $B_{a}$ of the target, as illustrated in Fig. 3. Consequently, when applied to GRD data, speckle tracking theoretically fails near the burst seams, and depending on the ice displacement, small gaps appear in the resulting displacement maps.

Azimuth frequency deramping and common band filtering extract the overlapping part of the Doppler spectra of corresponding burst (as opposed to consecutive bursts). These processing steps do not compensate for the fact that disjoint parts of the reflectivity spectra are observed with the antenna steered

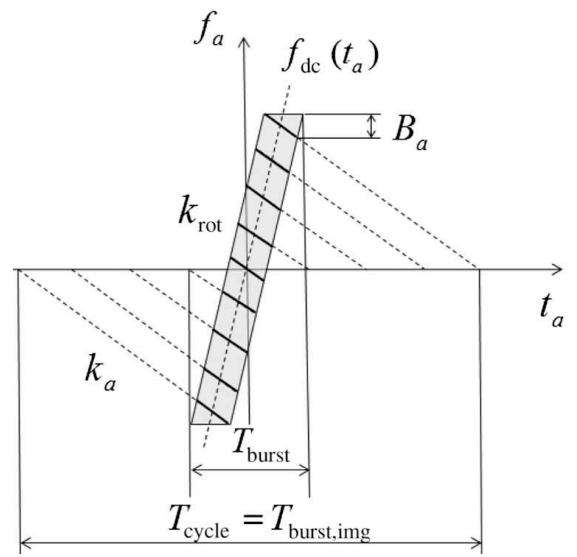

Figure 3. Time-frequency diagram of a TOPS azimuth line after time-domain unfolding [12]. 
backward and forward in consecutive bursts. The common band filtering compensates for slight ice displacements when corresponding bursts are combined, but it does not address motion across burst seams.

The decorrelation of speckle in consecutive bursts is one reason why the GRD product is not suited for speckle tracking. Another reason is that interpolation, upsampling, and common band filtering in practice call for a prior azimuth frequency de-ramping, which cannot be applied to the detected GRD product [12].

Fortunately, in the Sentinel-1 SLC product neighbouring bursts overlap [6], so patches from corresponding bursts can be cross-correlated, provided the azimuth displacement of the ice is not excessive. This is illustrated with the example in Fig. 4, where ice moves out of one burst, but is covered by the following burst in both data sets. All patches are fully covered by corresponding bursts if the azimuth ice displacement $\Delta_{a z}$ meets the requirement

$$
\Delta_{a z}<d_{o v l}-l_{p, a z}
$$

where $d_{o v l}$ is the overlap of consecutive bursts, and $l_{p, a z}$ is the azimuth patch dimension. In this case, gaps in the displacement maps can be avoided. Hence, when using SLC data, also speckle tracking is expected to work.

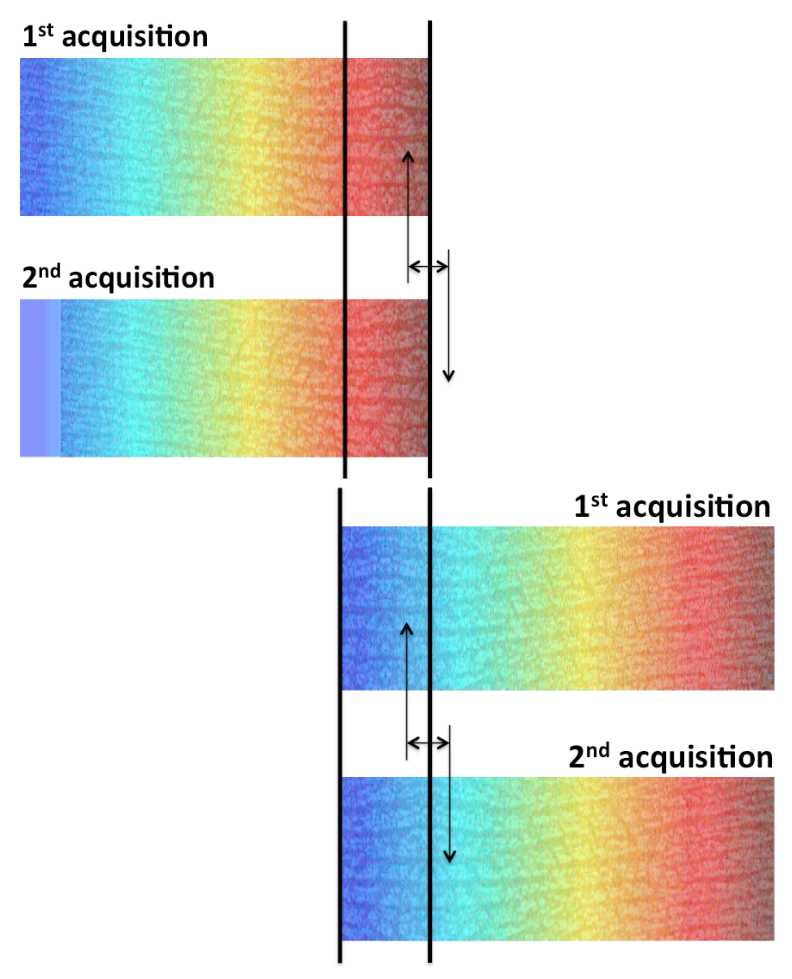

Figure 4. Bursts overlap in the SLC product
The aim of the study presented in this paper is to empirically verify the expectations just outlined. This is done by addressing questions such as:

- Which of the four multi-temporal cross-correlations that can be computed in the overlap areas are useful?

- Where do consecutive (multi-temporal) bursts decorrelate?

- Can the GRD product be expected to lead to more velocity gaps than the SLC product?

- Do the dual squint angles within the burst overlap provide valuable glaciological information?

\section{METHODOLOGY}

The analysis makes use of the operational interferometric post processing chain IPP. The IPP processor has been developed by the National Space Institute at the Technical University of Denmark (DTU Space). Originally it had only an interferometric capability, (double difference and DEM elimination), but recently it has been upgraded with an offset tracking capability in the frame of ESA's Climate Change Initiative. The IPP processor supports the Sentinel-1 SLC product. Initially, the IPP processor was tested with RADARSAT-2 data acquired in TOPS mode [13] to make sure that it was ready as soon as Sentinel-1 SLC data pairs from Greenland became available. Fig. 5 shows an example of an ice velocity map generated with the IPP processor.

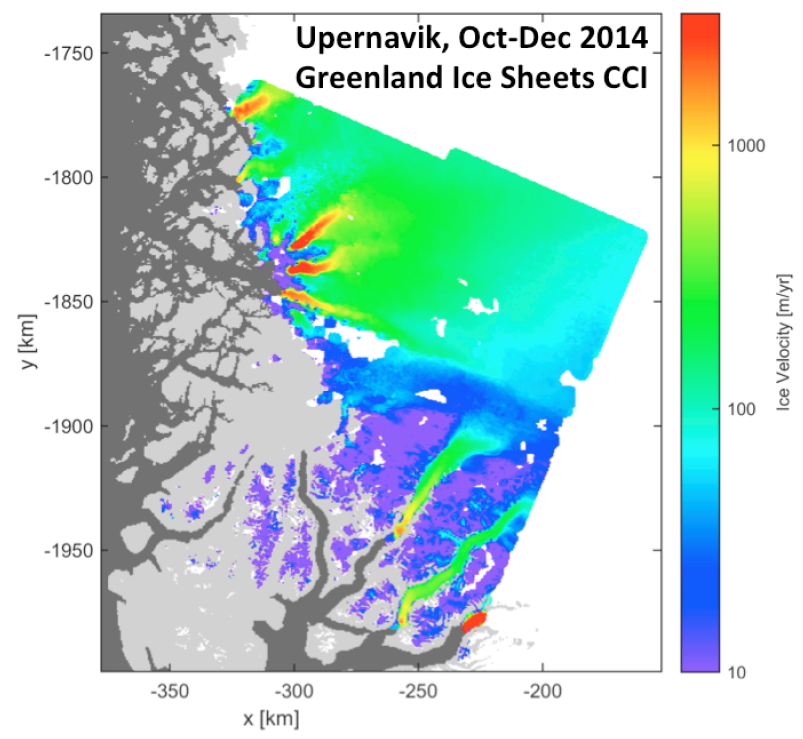

Figure 5. Ice velocity map [m/year] of the Upernavik region in West Greenland generated from Sentinel-1A SLC data using the IPP processor. The gray areas are masked out, as they are ice free. 
The offset tracking capability of the IPP processor is based on the normalized cross-correlation function

$$
\operatorname{NCC}(i, j)=\frac{\sum_{k, l}\left(s(i+k, j+l)-\mu_{s}\right)\left(r(k, l)-\mu_{r}\right)}{\sum_{k, l}\left|s(i+k, j+l)-\mu_{s}\right|^{2} \sum_{k, l}\left|r(k, l)-\mu_{r}\right|^{2}}
$$

Within the burst overlaps the IPP processor computes the NCC for both pairs of corresponding bursts shown in Fig. 4. The resulting displacement estimates are computed as a weighted average of the valid displacement estimates (two or less). A displacement estimate is valid if it exceeds the threshold defined for a set of quality parameters including

- the magnitude of the NCC peak and

- the signal-to-noise ratio (SNR).

In this context the SNR is defined as the ratio of the NCC peak to the average magnitude of the NCC function outside a 5 pixels by 5 pixels window centered at the NCC peak.

For an ice patch that is only included in one of the two corresponding bursts (see the upper half of Fig. 4) the displacement estimate is likely to be deemed invalid. In this case the weighted average of displacement estimates degenerates to the estimate provided by the burst pair in the lower half of Fig. 4.

The IPP processing chain does not exploit the two (multi-temporal) cross-correlations of consecutive bursts, one of which is illustrated in Fig. 6. However, in this study, the IPP was run in such a way that it also provided this cross-correlation of consecutive bursts.

When the GRD product is used for offset tracking, large azimuth displacements across the fixed burst seams lead to a cross-correlation of consecutive bursts like in Fig. 6, so basically the figure also illustrates the usage of GRD data (though deramping and azimuth common band filtering cannot be applied to GRD data).

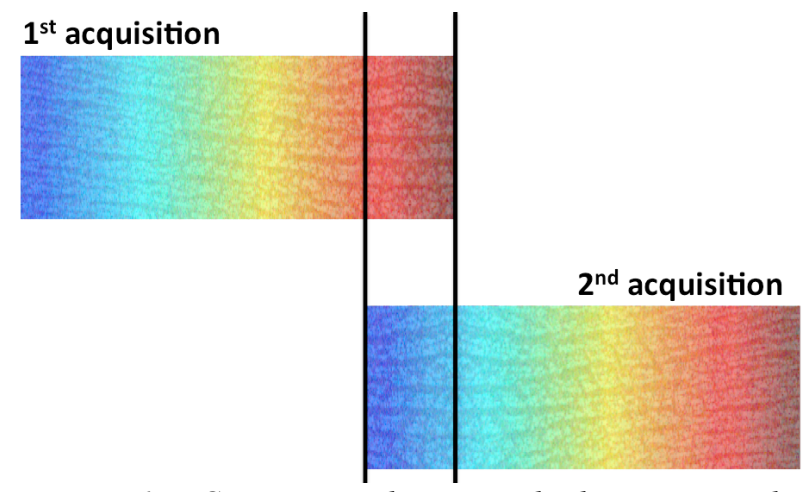

Figure 6. Consecutive bursts with disjoint Doppler spectra within the spatial overlap.

\section{RESULTS}

The results are obtained with the Upernavik scene $\left(72^{\circ} 47^{\prime} \mathrm{N}, 56^{\circ} 09^{\prime} \mathrm{W}\right)$. While the velocity map in Fig. 5 is based on five pairs of acquisitions, only one pair is used in the following (acquisition dates: 2014.11.15 and 2014.11.27).

The magnitude image is shown in Fig. 7. The bursts are properly mosaicked (without doubling the overlap regions), whereas the three sub-swaths (IW1-3) are not seamlessly combined. Basically the scene is composed of the ice sheet in the upper right, the sea in the lower left, and in between an ice-free area. Also, several glaciers are seen in the upper part of IW2 and in the lower part of IW1.

A 12-days azimuth displacement map is shown in Fig. 8. In areas where bursts overlap, the displacement estimates resulting from cross-correlating consecutive bursts are laid over the standard IPP output. The size of the detected patches is 256 pixels by 64 pixels, but the output grid spacing is 40 pixels by 10 pixels (range by azimuth). This means that no estimates exist near the edges in the overlap areas, where zeros are inserted instead. In Fig. 8 this is seen as two green lines framing each overlap area.

The displacements are generally in the sub-pixel level, but for the glaciers they are up to about 10 pixels. Not surprisingly the sea and the fjords are characterized by erroneous displacement estimates. Areas with erroneous displacements stand out as a random pattern of mainly blue and red pixels, because without a proper NCC peak, the displacement estimates are spread over the entire \pm 20 pixel range of the colour map. Apart from a few small ice-covered areas near the ice edge, offset tracking seems to work fine when based on corresponding bursts, but when estimated from consecutive bursts (the overlaid strips), it fails over most of the ice sheet. Feature tracking is likely to fail because there are no features, and speckle tracking is supposed to fail because there is no spectral overlap between consecutive bursts. The good performance over the glaciers and the ice-free areas is probably because features are present there.

Fig. 9 shows an NCC map. As expected, the crosscorrelation is low over the sea and over the fjords, but high over the ice-free (but hardly snow-free) areas. Over the ice sheet the NCC varies considerably, probably due to temporal decorrelation caused by melt events (at low altitudes), local snow accumulation, and aeolian processes. Also ice flow induced decorrelation is seen, e.g. above the middle of IW1. Again, the overlaid strips are the result of cross-correlating consecutive bursts. In accordance with Fig. 8, the NCC is very low over the featureless ice sheet, where speckle tracking of consecutive bursts fails. 


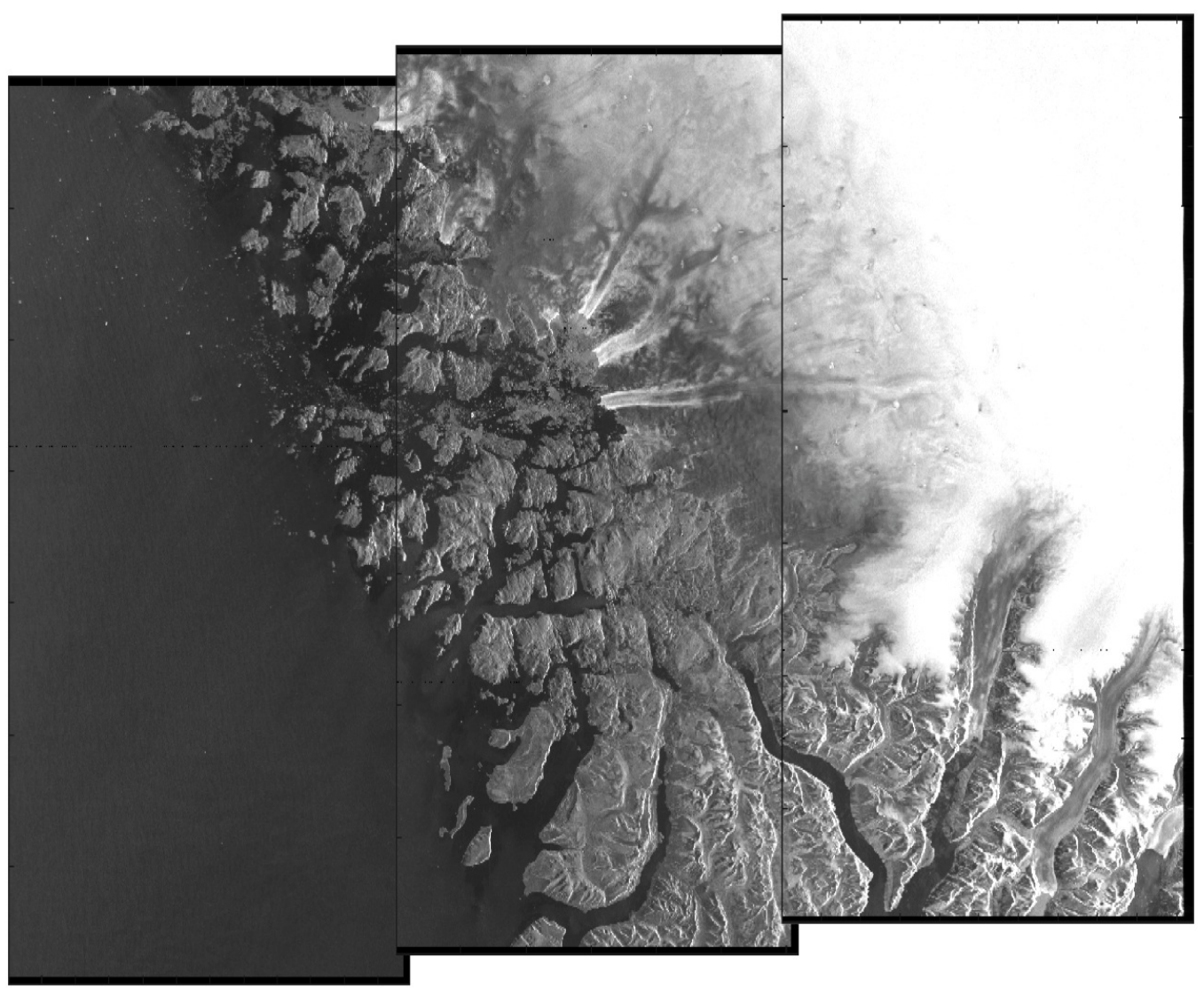

Figure 7. Upernavik scene: Magnitude.

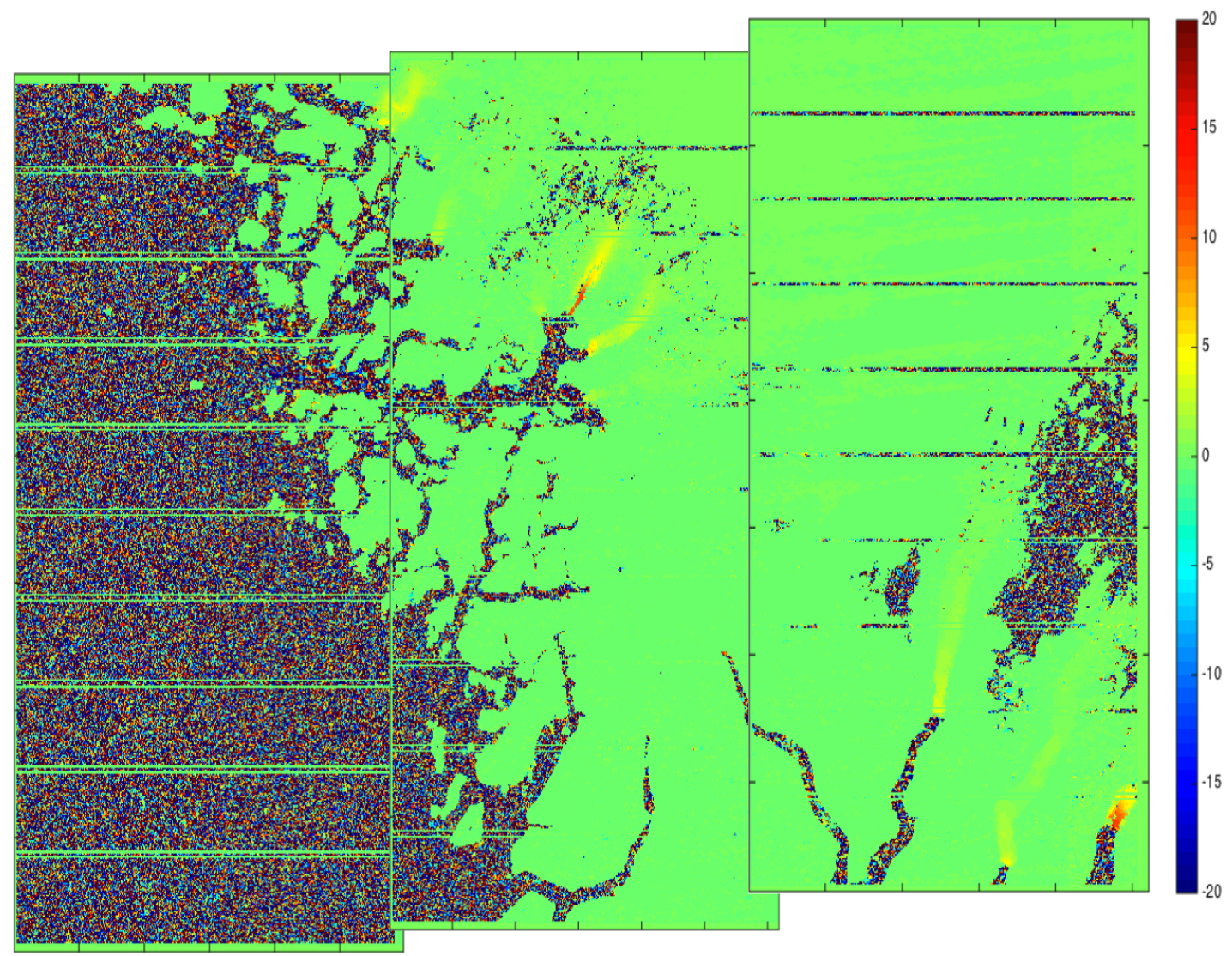

Figure 8. Upernavik scene: Azimuth displacement map. Units of colour bar: pixels. 


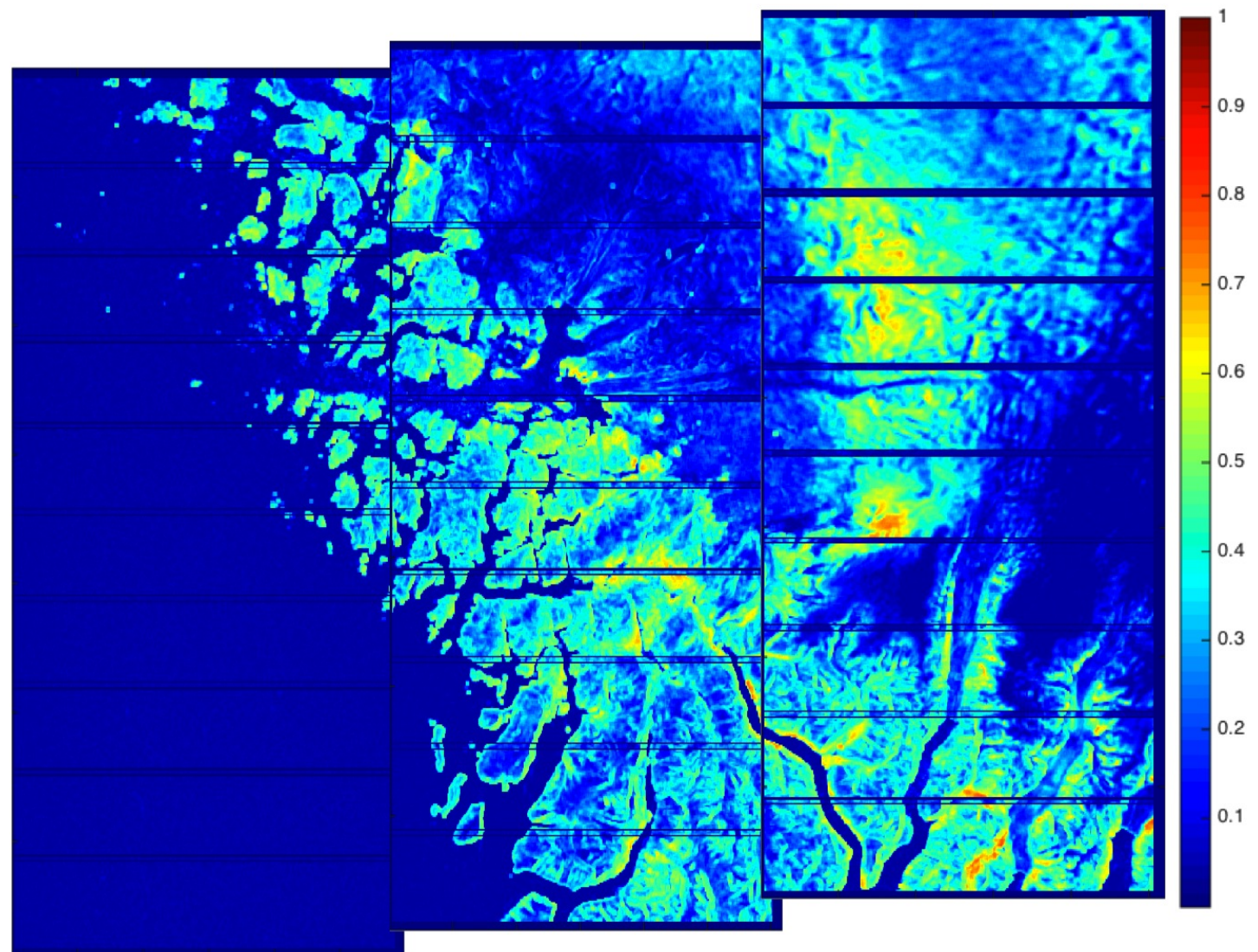

Figure 9. Upernavik scene: Maximum of normalized cross-correlation (NCC).

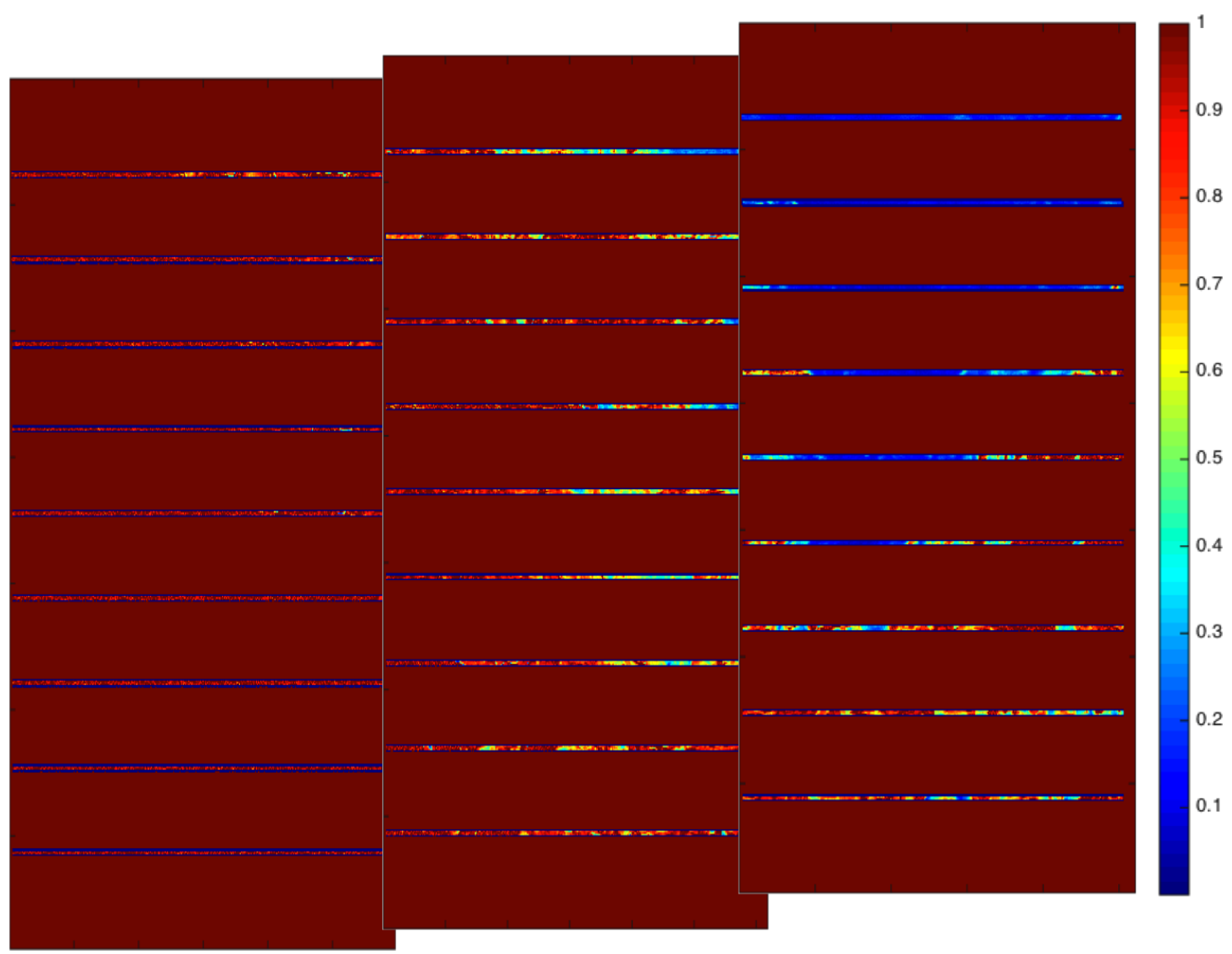

Figure 10. Upernavik scene: Relative NCC max (consecutive bursts / corresponding bursts). 
Fig. 10 shows the ratio of the NCC with and without the overlay strips. Outside the overlap areas, the two images are identical and the ratio is unity, but within the overlap areas the figure shows how much the NCC is reduced when switching from corresponding bursts to consecutive bursts. As expected, the relative reduction is largest over the ice sheet, where speckle tracking of consecutive bursts fails. Over the ice-free areas the reduction is less significant, presumably because the NCC is ensured by features. Over the sea, the ratio can exceed unity because the scene completely decorrelates, both for corresponding and consecutive bursts. Fig. 10 is very similar to the corresponding SNR ratio map, and both figures indicate the impact of features on the NCC.

\section{CONCLUSIONS}

Offset tracking has been applied to a Sentinel-1A scene from Upernavik, Greenland. The usual crosscorrelation of corresponding bursts works fine for most of the scene. When consecutive bursts are crosscorrelated in the overlap areas, offset tracking fails over the ice sheet. It works well over the ice-free areas, though the NCC and SNR quality parameters are reduced. These observations are as expected: 1) The ice sheet is mostly featureless, and within the spatial overlaps, speckle tracking is supposed to fail because consecutive bursts do not overlap in the spectral domain. 2) The glaciers and the ice-free areas are rich in features, which ensure a successful offset tracking, though the quality parameters are lower because speckle does not contribute to the cross-correlation peak and/or the features depend somewhat on the squint angle.

With the GRD product, consecutive bursts are crosscorrelated whenever the azimuth displacement exceeds the size of the patches used, and hence speckle tracking results in gaps in the velocity maps. The SLC product is applicable for feature tracking and also for speckle tracking provided the azimuth displacement is smaller than the burst overlap minus the patch size. This requirement is likely met because the ice velocity tends to be small where the ice is featureless.

\section{REFERENCES}

[1] E. Rignot, J. Mouginot, B. Scheuchl, "Ice flow of the Antarctic ice sheet", Science, Vol. 333, p. 1427-1430, 2011.

[2] R. Michel, E. Rignot, "Flow of Moreno glacier, Argentina, from repeat-pass Shuttle Imaging Radar images: comparison of the phase correlation method with radar interferometry", Journal of Glaciology, Vol. 45, No. 149, p. 93-100, 1999.

[3] R. De Lange, A. Luckman, T. Murray, "Improvement of satellite radar feature tracking for ice velocity derivation by spatial frequency filtering”, IEEE Transactions on Geoscience and Remote Sensing, Vol. 45, p. 2309-2317, 2007.

[4] A.L. Gray, N. Short, K.E. Mattar and K.C. Jezek, "Velocities and flux of the Filchner ice shelf and its tributaries determined from speckle tracking interferometry", Canadian Journal of Remote Sensing, Vol. 27, No. 3, 2001.

[5] I. Joughin, "Ice-sheet velocity mapping: a combined interferometric and speckle-tracking approach", Annals of Glaciology, Vol. 34, No. 1, p. 195-201, 2002.

[6] https://sentinel.esa.int/web/sentinel/userguides/sentinel-1-sar/product-types-processinglevels/level-1

[7] F. De Zan, A.M. Guarnieri, "TOPSAR: Terrain observation by progressive scans", IEEE Transactions on Geoscience and Remote Sensing, Vol. 44, No. 9, p. 2352-2360, 2006.

[8] http://www.esa.int/spaceinvideos/Videos/2014/03/ Mike_Oldfield_sends_greeting_to_ESA and the Sentinel team

[9] https://sentinel.esa.int/web/sentinel/userguides/sentinel-1-sar/acquisitionmodes/interferometric-wide-swath

[10] N. Miranda, S1 space project team, S1 PDGS team https://earth.esa.int/documents/10174/669756/Sent inel-1 System Data.pdf/a3a56776-9cbb-487a94a9-11bd20579d79, Presented at PolInSAR 2013, ESRIN, 2013.

[11] P Prats-Iraola, R. Scheiber, L. Marotti, S. Wollstadt, A.. Reigher, "TOPS interferometry with TerraSAR-X”, ", IEEE Transactions on Geoscience and Remote Sensing, Vol. 50, No. 8, p. 3179-3188, 2012.

[12] R. Scheiber, M. Jager, P. Prats-Iraola, F. De Zan, D. Geudtner, "Speckle tracking and interferometric processing of TerraSAR-X TOPS data for mapping nonstationary scenarios", IEEE Journal of Selected Topics in Applied Earth Observations and Remote Sensing, DOI 10.1109/JSTARS.2014.2360237, 2014.

[13] D. Geudtner, "Implementation of the TOPS mode on RADARSAT-2 in support of the Copernicus Sentinel-1 mission; RADARSAT-2 TOPS SAR interferometry (InSAR) scene pair data acquisitions", ESA reference S1-TN-ESA-SY-0452, 2014. 